\title{
Longitudinal Guided Wave Inspection of Small-Diameter Elbowed Steel Tubes with a Novel Squirrel-Cage Magnetostrictive Sensor
}

\author{
Yao Liu, ${ }^{1}$ Xiucheng Liu $\mathbb{D}^{1},{ }^{1}$ Chehua Yang, ${ }^{2}$ Wenxin Guo, ${ }^{3}$ Bin $\mathrm{Wu},{ }^{1}$ and Cunfu $\mathrm{He}^{1}$ \\ ${ }^{1}$ College of Mechanical Engineering and Applied Electronics Technology, Beijing University of Technology, Beijing, China \\ ${ }^{2}$ Graduate Institute of Manufacture Technology, National Taipei University of Technology, Taipei, China \\ ${ }^{3}$ Beijing Pansmart Pipeline Technology Co., Ltd, Beijing, China
}

Correspondence should be addressed to Xiucheng Liu; xiuchliu@bjut.edu.cn

Received 15 July 2019; Revised 8 October 2019; Accepted 19 October 2019; Published 12 November 2019

Academic Editor: Salvatore Surdo

Copyright (C) 2019 Yao Liu et al. This is an open access article distributed under the Creative Commons Attribution License, which permits unrestricted use, distribution, and reproduction in any medium, provided the original work is properly cited.

In the study, ultrasonic longitudinal mode guided waves were employed to detect defects in elbowed tubes (without welds) with a diameter of $10 \mathrm{~mm}$. Finite element simulation results highlighted that the emitted $\mathrm{L}(0,1)$ mode guided waves experienced strong reflection and mode conversion at the elbow region to generate $\mathrm{F}(1,1)$ mode, followed by slow and weak $\mathrm{F}(2,1)$ mode. The guided wave reflected from the elbow with a through-wall defect was manifested as two overlapped wave packets, which were good indicators of a defective elbow. To conduct $\mathrm{L}(0,1)$ mode guided waves inspection on the small-diameter elbowed tubes, a novel tailored squirrel-cage magnetostrictive sensor was employed in the experiment. The new sensor employed the configuration of segmental iron-cobalt strips and small-size permanent magnet arrays. The entire sensor is composed of two identical C-shaped sensor elements and can be recycled and installed conveniently. Experimental results obtained from healthy and defective tubes were consistent with the conclusions obtained from finite element simulations. An artificial through-wall defect at the elbow and a notch defect at the straight part of the tube could be simultaneously detected by $\mathrm{L}(0,1)$ mode guided waves through comparing experimental signals with simulation results.

\section{Introduction}

Metallic elbowed tubes with a small diameter (less than $20 \mathrm{~mm}$ ) are crucial parts for transporting waste heat, fuel oil, water coolant, etc., among power equipment of ships [1]. Corrosions generated at the tubes during long-term service are hidden dangers and will evolve to through-wall defects causing the leakage of the transported medium. The leakage of medium transported in the tubes may lead to sudden shutdown of power equipment. For the safety of power equipment and reduce the probability of accidents, it is necessary to carry out the corrosion condition assessment of smaller-diameter tubes. Nondestructive testing (NDT) technologies including remote field eddy current $[2,3]$, magnetic flux leakage [4-6] (only applicable to ferromagnetic tubes), and ultrasound $[7,8]$ have been applied to detect defects in small-diameter metallic tubes. Ultrasonic guided waves (UGWs) technology is another candidate option for defect inspection in small-diameter elbowed metallic tubes.
The UGWs of optimally selected modes and frequencies can propagate along the tested tubes for a long distance to interact with the defects in a tube. The UGWs reflected from the defects can be detected with a proper sensor, and the obtained voltage signal could indicate the locations and sizes of the defects. The performances of UGWs in evaluating the corrosion in large-diameter pipelines [9-11] and welding elbows $[12,13]$ have been extensively explored. However, the applications of UGWs in metallic elbowed tubes with a small diameter of $10 \mathrm{~mm}$ were seldom reported.

In this study, the feasibility of applying longitudinal guided waves in the inspection of elbowed tubes (without welds) with a diameter of $10 \mathrm{~mm}$ was investigated. Finite element simulation was performed to highlight the inherent reflection of $\mathrm{L}(0,1)$ mode and the mode conversion of UGWs at a $90^{\circ}$ elbow with and without defects. A novel squirrel-cage magnetostrictive sensor (MsS) was tailor-made for smalldiameter tubes to generate and receive $\mathrm{L}(0,1)$ mode UGWs. The performances of the novel squirrel-cage MsS in detecting 
artificial defects in small-diameter elbowed tubes were experimentally explored. The experimental results were consistent with the conclusions obtained from finite element simulation results, indicating that the through-wall defect at the elbow and a notch defect at the straight part could be simultaneously detected by $\mathrm{L}(0,1)$ mode UGWs.

In Section 2, finite element simulations were performed to investigate the propagation behavior of UGWs in smalldiameter elbowed tubes. In Section 3, the design details of squirrel-cage MsS were given and the central frequency of the sensor was experimentally determined. In Section 4, the UGW inspection results obtained from defective smalldiameter elbowed tubes were discussed by comparing the experimental and finite element simulation results. The conclusions are drawn in Section 5.

\section{Longitudinal Guided Waves in Small- Diameter Elbowed Tubes}

The ultrasonic guided waves propagating in tubes exist in the forms of multiple modes (longitudinal, flexural, and torsional modes). The propagation behaviors of the UGWs modes in a straight tube can be simply investigated using the dispersion curves displaying the dependency of phase (or group) velocity, $V_{\mathrm{p}}$ (or $V_{\mathrm{g}}$ ), on the frequencies. DISPERSE software is employed to calculate the dispersion curves (see Figure 1(a)) of the longitudinal and flexural modes in a hollow cylinder of steel (Young's modular $E=216.9 \mathrm{GPa}$, Poisson's ratio $v=0.286$, and density $\rho=7.9 \mathrm{~kg} / \mathrm{m}^{3}$ ) [14]. The inner and outer diameters of the hollow cylinder are $6 \mathrm{~mm}$ and $10 \mathrm{~mm}$, respectively.

To simplify the identification process of UGWs signals obtained from the defective tube, the frequency range with less UGWs modes is generally selected and the UGW modes experiencing weak dispersion is preferred. In the frequency range lower than $180 \mathrm{kHz}$, as sketched in Figure 1(a), only two modes can be generated. It is more difficult to generate pure flexural mode compared to longitudinal modes due to the difficulties in the sensor design [15]. Therefore, the lowest-order longitudinal mode referred as $\mathrm{L}(0,1)$ was selected for defect detection in the investigated smalldiameter tube. The operation frequencies of $\mathrm{L}(0,1)$ mode were lower than $150 \mathrm{kHz}$ in order to suppress the intrinsic mode dispersion.

In Section 3, a magnetostrictive sensor (MsS) employing single solenoid coil wound by enameled copper wires of $0.35 \mathrm{~mm}$ in diameter was designed to generate $\mathrm{L}(0,1)$ mode in the small-diameter tube. The central frequency, $f_{c}$, of the MsS could be roughly adjusted by changing the width, $w$, of the excitation solenoid coil. The reported empirical equation $\left(w=0.5 V_{\mathrm{p}} / f_{\mathrm{c}}\right)$ indicated that the coil width should be equal to the half-wavelength $(\lambda / 2)$ of UGWs at the expected central frequency [16]. Based on the dispersion curves in Figure 1(a), the relationship between the half-wavelength of UGWs and the frequency was calculated, as shown in Figure 1(b). If the central frequency of the MsS is expected to be around $100 \mathrm{kHz}$, the width of solenoid coil is estimated to be around $25.4 \mathrm{~mm}$ (Figure 1(b)).
Finite element simulation was conducted in the commercial ABAQUS software to reveal the propagation behaviors of $\mathrm{L}(0,1)$ mode in the small-diameter elbowed tube. The modeled elbowed tube was identical to the tube tested in subsequent experiments. The tube has an outer diameter of $10 \mathrm{~mm}$ and a wall thickness of $2 \mathrm{~mm}$. As sketched in Figure 2, the entire tube is elbowed $90^{\circ}$ and the lengths of the horizontal and vertical straight section are $1.105 \mathrm{~m}$ and $0.815 \mathrm{~m}$, respectively. The radius of the centerline of the elbow is $15 \mathrm{~mm}$. A cylinder with a diameter of $5 \mathrm{~mm}$ was subtracted from the outer elbow wall to form a simulated through-wall defect. In the horizontal section of the tube, a simulated notch defect with a depth of $1.8 \mathrm{~mm}$ and a width of $1 \mathrm{~mm}$ was prepared. The locations of the two simulated defects in the tube are illustrated in the upper right inset of Figure 2.

The entire mode is meshed with free hexahedron, and the mesh refinement is applied in the elbow region and the region around the notch defect. The operation of mesh refinement realizes $0.3 \mathrm{~mm}$ in mesh element size and the rest of the regions of the mode have a mesh element size of $0.5 \mathrm{~mm}$. Pressure of $10 \mathrm{MPa}$ in the form of five-cycle sinusoidal tone burst modulated by a Hanning window with a central frequency of $90 \mathrm{kHz}$ was assigned to all the nodes at the cross-section area of the upper tube end to generate $\mathrm{L}(0,1)$ mode in the tube.

The snapshot pictures shown in Figure 2 are taken from different times during the propagation of $\mathrm{L}(0,1)$ mode in a healthy elbowed tube. When the emitted UGWs encounter the elbow, partial energy of the UGWs is reflected back to the upper end and the mode conversion from $\mathrm{L}(0,1)$ mode to $\mathrm{F}(1,1)$ mode can be confirmed. It can be observed that after the $\mathrm{F}(1,1)$ mode, an anomalous wave is generated at the elbow. Both the amplitude and velocity of the anomalous waves are lower than those of the main reflection or transmission wave. The group velocity of the anomalous waves is estimated as around $1200 \mathrm{~m} / \mathrm{s}$, which is quite close to the group velocity of the $\mathrm{F}(2,1)$ mode. Therefore, we tend to believe that the high-order flexural mode of $\mathrm{F}(2,1)$ mode that was generated during the mode conversion process occurred at the elbow.

To clarify the mode conversion and reflection of UGWs at the elbow region, two circumferences, respectively, marked as " $A$ " and " $B$ " of the tube were selected as the data extraction sources. The locations of the two circumferences are shown in Figure 3(a). The time-dependent displacement extracted from A (or B) circumference was treated as the UGWs emitting into (or passing through) the elbow.

The UGW signals received from four points at A circumference are plotted in Figure 3(b). The second wave packet in the received signals represents the $\mathrm{L}(0,1)$ mode reflected from the elbow. At the top and bottom points of A circumference, $F(1,1)$ mode was not included in the reflected waves. However, the reflection of $F(1,1)$ mode could be observed from the results extracted from the points at the left (outward) and right (inward) wall. Therefore, it was inferred that the mode conversion from $\mathrm{L}(0,1)$ mode to $\mathrm{F}(1,1)$ mode mainly occurred at the surface whose normal curvature experienced sudden changes [17]. 


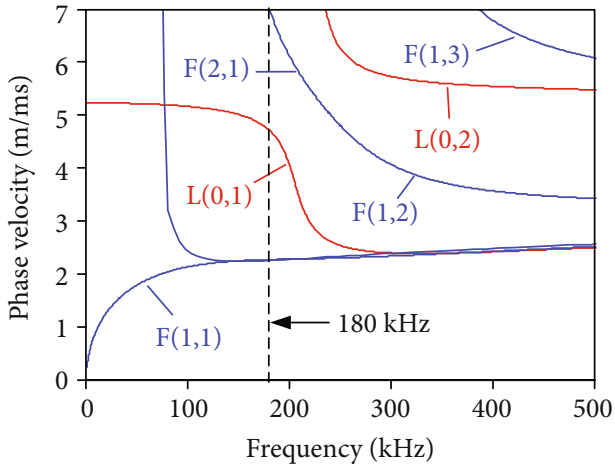

(a)

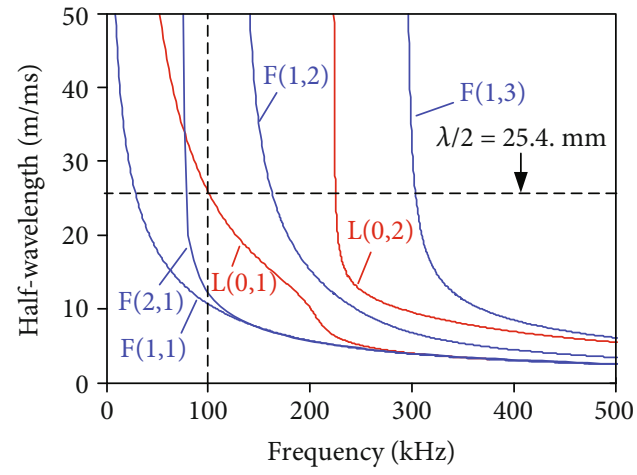

(b)

Figure 1: (a) Dispersion curves of longitudinal and flexural modes obtained from the investigated tube and (b) dependencies of halfwavelength of UGWs on the frequency.

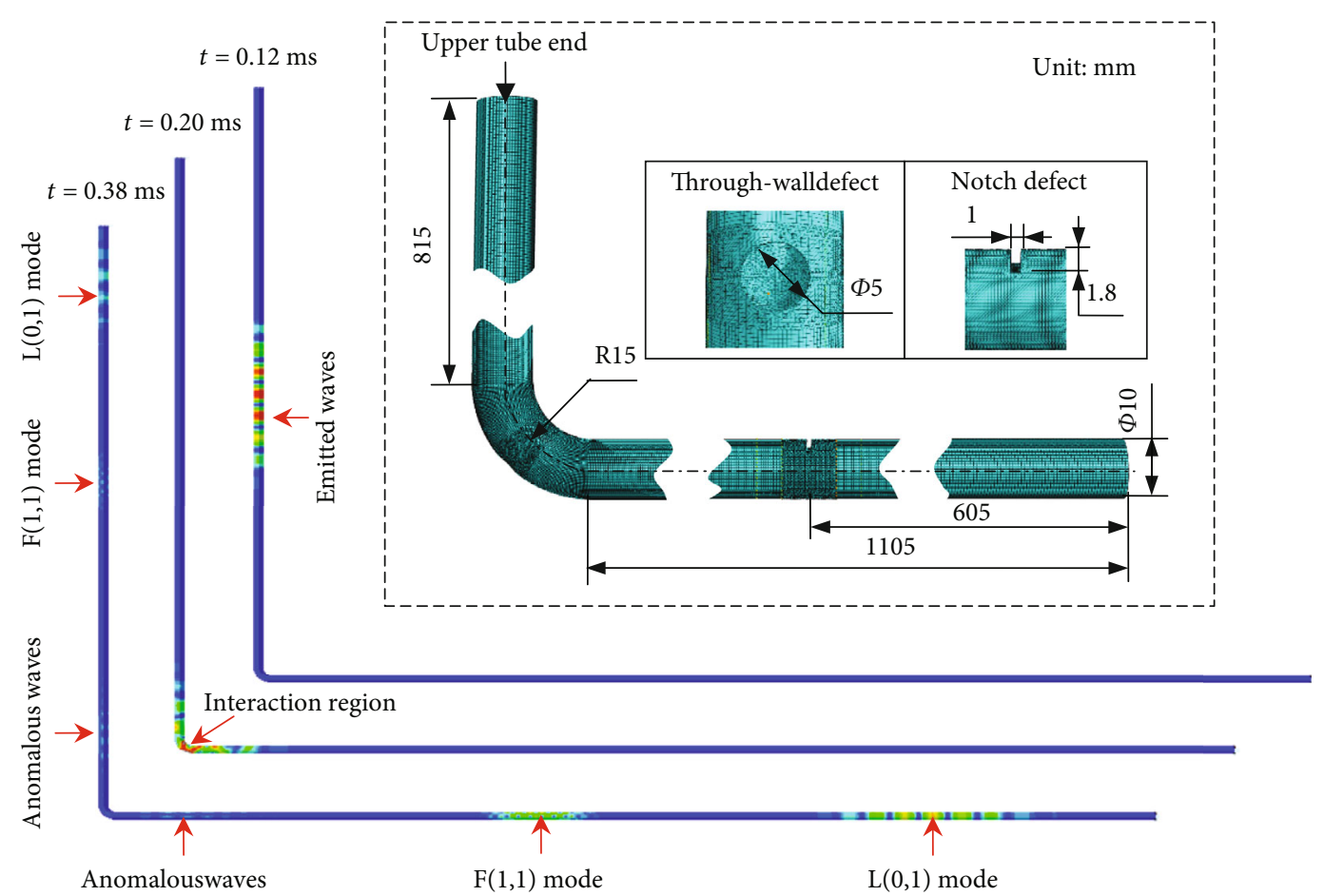

FIGURE 2: Finite element simulation of the propagation of UGWs in the investigated small-diameter elbowed tube without defects. The upper right inset shows the mesh results and geometrical sizes of the entire model.

The inherent UGW mode conversion at the elbow induced regular wave packets in the UGW inspection signals to disturb the defect signal reorganization. To suppress the $\mathrm{F}(1,1)$ mode, the signals received along the A and B circumferences were superposed. After the amplitude of the superposed signals being divided by four, the emitted and reflected $\mathrm{L}(0,1)$ modes were shown in Figures $3(\mathrm{c})$ and $3(\mathrm{~d})$. Thus, the transmission and reflection of pure $\mathrm{L}(0,1)$ mode at the elbow region can be presented to provide prior knowledge for experimental result analysis. The energy reflection efficiency, $\beta$, of the $\mathrm{L}(0,1)$ mode at the elbow was estimated with the ratio of the first reflection wave energy to the emitted wave energy. The energy of the wave packets was calculated by the summation of the square of the wave packets, as shown in the upper chart of Figure 3(c). The value of $\beta$ was estimated to be around $7.4 \%$.

Figure 3(d) shows the simulated UGW signal reflected from and passing through the elbow with a through-wall defect. The reflection UGW signal was manifested as two overlapped wave packets. The components of the overlapped wave packets were explored by comparing experimental results with simulation results in Section 4. The significant differences in the waveform between the signals, respectively, reflected from the healthy and defective elbow regions indicated that the $\mathrm{L}(0,1)$ mode could be used to detect the through-wall defect in the elbow. 


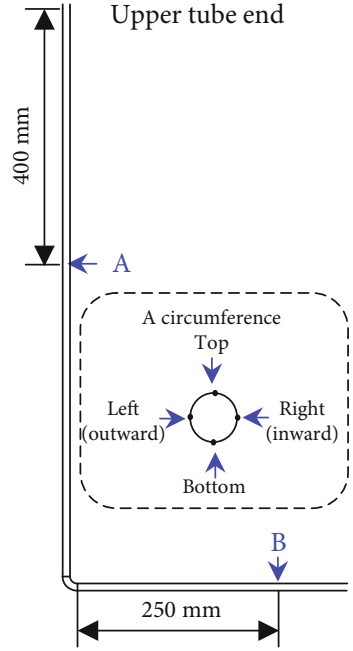

(a)

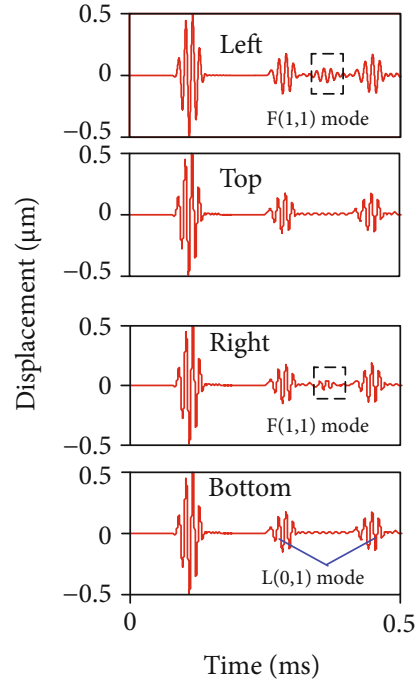

(b)

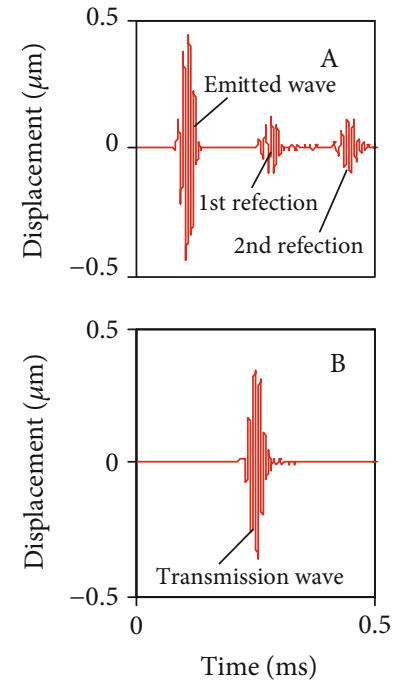

(c)
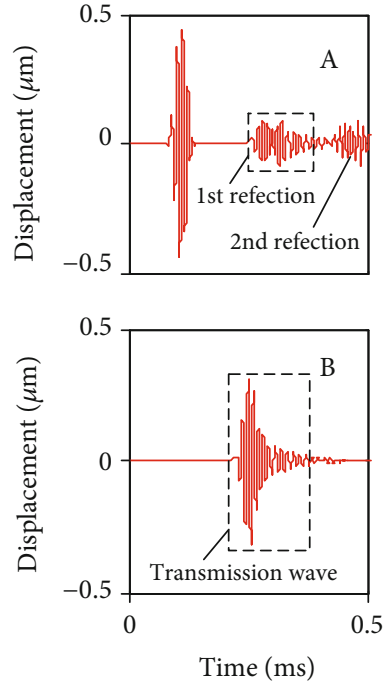

(d)

FIGURE 3: Simulation results of the UGWs extracted from the locations at both sides of the elbow. (a) Locations of the data extraction sources in the investigated tube. (b) Results extracted from the A circumference. (c, d) Results obtained from the models of the healthy and defective elbows.

\section{Design of a Squirrel-Cage Magnetostrictive Sensor}

Magnetostrictive sensor is a favorable option in the construction of ultrasonic guided wave inspection system due to its flexibility in the configuration design of various types of structures. Several types of MsSs had been developed to generate longitudinal mode UGWs in steel rod and strand with small nominal diameters based on the inherent magnetostrictive effects of the tested ferromagnetic materials [1820]. However, the electro-ultrasonic energy transfer efficiency of the MsS is limited because the inherent magnetostriction of steel is weak. To improve the energy transfer efficiency of MsS, smart materials with high magnetostriction such as nickel [21], iron-cobalt alloy [22, 23], and Galfenol flakes-polymer composite [24] were used as the electroultrasonic energy transfer element and usually pasted onto the surface of the tested structures.

The utilization of magnetostrictive strip or patch allows the guided wave inspection in nonferromagnetic waveguide with MsS. A novel design of magnetostrictive patch sensor for inspecting large-size pipelines and plates had been reported $[25,26]$. However, the magnetostrictive patch sensor for detecting longitudinal mode UGWs in smalldiameter tubes was seldom explored. The difficulty in the design of magnetostrictive patch sensor for a tube with a diameter of $10 \mathrm{~mm}$ mainly lies in wrapping the magnetostrictive strip or patch into the shape of a cylindrical shell due to the stiffness of the strip or patch.

A tailored MsS based on a new configuration of segmental iron-cobalt patches and small-size permanent magnet arrays is proposed to generate and receive $\mathrm{L}(0,1)$ mode UGWs in the tested small-diameter elbowed tubes. Inspired by the squirrel-cage motor, we designed the new sensor configuration. As shown in Figure 4(a), the entire sensor is com- posed of two identical C-shaped sensor elements which can facilitate reusage and installation.

A total of seven biased magnetostrictive patches with identical dimensions of $20 \mathrm{~mm}$ (length) $\times 1.5 \mathrm{~mm}$ (width) $\times$ $0.15 \mathrm{~mm}$ (height) were evenly distributed around the circumferential direction and assembled onto the plastic holder of each C-shaped sensor element. The dimensions of ferrite permanent magnets is $3 \mathrm{~mm}$ (length) $\times 2 \mathrm{~mm}$ (width) $\times 1$ $\mathrm{mm}$ (height), and its magnetization axis is parallel to the length direction. Two ferrite permanent magnets were placed at two ends of an individual magnetostrictive patch to provide a static magnetic field for patch magnetization.

To examine the distribution of static magnetic field in the magnetostrictive patch, the physical model shown in Figure 4(a) was analyzed by finite element simulation in COMSOL platform. In the simulation, the relative permeability of the patch was assigned as 2000. Due to the axial symmetry in the magnetizer, the distributions of the magnetic field in all the patches were the same. Therefore, the result of magnetic induction intensity, $B$, was taken from one of the fourteen patches. Figure 4(b) shows the normalized profile of $B$ extracted from the center line of the patch along its length direction and the inset shows the distribution of $B$ at the transverse cross-section of the patch.

Though the magnetic flux intensity extracted at both endpoints of the patch was smaller than that of the rest locations, the normalized value of $B$ was changed to around 0.916 and the change rate was in the range of $\pm 9.17 \%$. Thus, the static magnetic field parallel to the axial direction of tube was thought to be evenly distributed in the patch. A solenoid coil with a width of $25 \mathrm{~mm}$ was wrapped around the plastic holder of the squirrel-cage frame. The coil is made of enameled copper wire and has 180 coil turns. The alternative current with a central frequency higher than $20 \mathrm{kHz}$ was fed into the coil to provide dynamic magnetic field parallel to the 


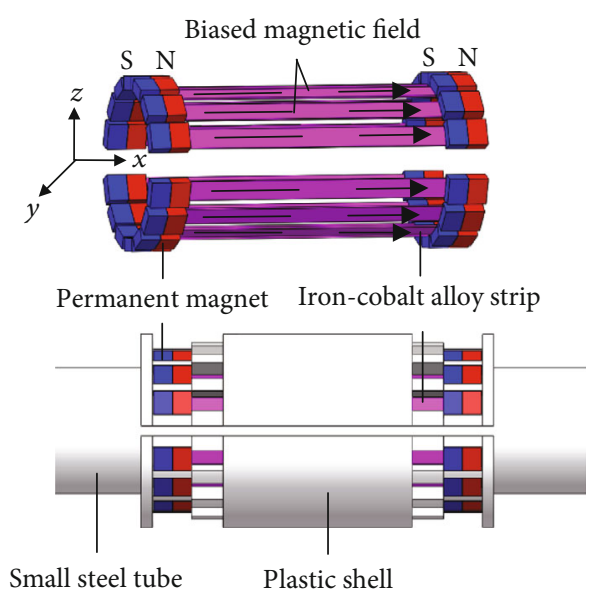

(a)

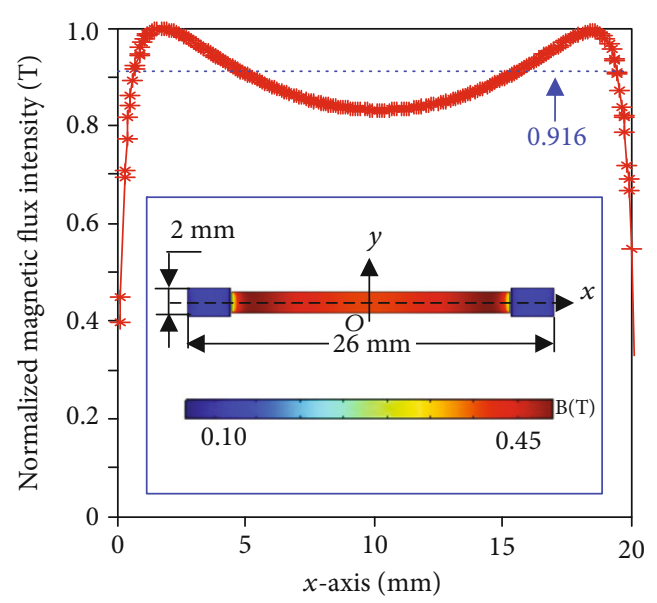

(b)

FIGURE 4: (a) Schematic diagram of the squirrel-cage element of MsS. (b) Simulated distribution of static magnetic field in a magnetostrictive patch.

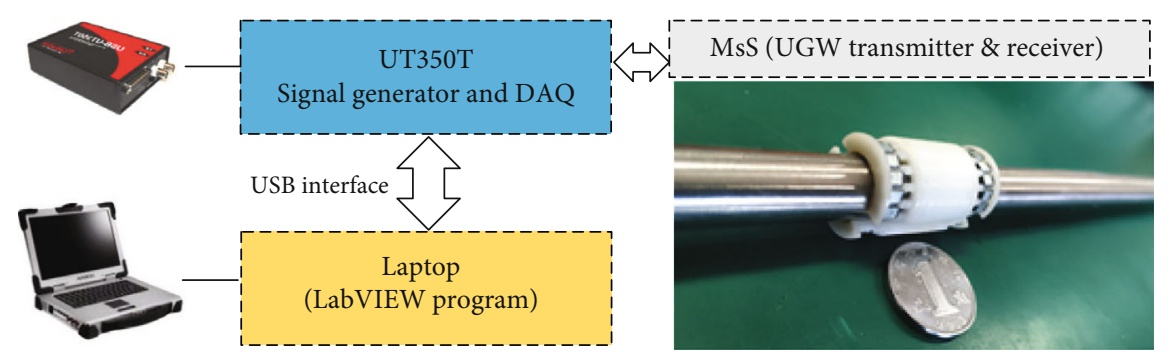

FIGURE 5: Experimental setup utilizing the proposed squirrel-cage MsS for UGW inspection of small-diameter tubes.

length direction of the strip. Governed by the magnetostrictive effect, ultrasonic longitudinal waves were generated in the patch under the interaction between the static and dynamic magnetic fields.

Figure 5 shows the experimental setup for UGW inspection of the tube. The squirrel-cage frame was attached onto the surface of the tested tube with epoxy. A portable device of UT350T (US Ultratek) was used as the signal generator and data acquisition module. The portable device was controlled by LabVIEW program in a laptop. One-cycle square wave with peak-to-peak amplitude of $250 \mathrm{~V}$ was emitted by the UT350T device and then fed into the excitation coils of the MsS. Ultrasonic longitudinal waves generated in all the fourteen strips transmitted into the tube through the epoxy couplant and propagated along the tube to form the longitudinal mode UGWs. According to the principle of inverse magnetostrictive effect, the UGWs reflected from the tube disturbed the static magnetic field in the strip so as to induce the voltage in the coil.

The central frequency of the squirrel-cage MsS in the pulse-echo mode was tested after installing the sensor at one end of a straight tube with a length of $1 \mathrm{~m}$. The inner and outer diameters of the tube are identical to those of the modelled tube in finite element simulation. The central frequency of the one-cycle square wave swept from $60 \mathrm{kHz}$ to $150 \mathrm{kHz}$ with a step of $10 \mathrm{kHz}$. Figure 6(a) shows typical waveforms of the multiple echoed waves reflected from the far end of the tube under the changing frequency. The peak-to-peak amplitude, $A$, of the first wave packet was estimated to draw the dependency of parameter $A$ on the operation frequency. As shown in Figure 6(b), the central frequency of the proposed MsS is around $90 \mathrm{kHz}$. By dividing the tube length by the time interval between the first two adjacent echoed waves, the propagation velocity of the UGWs in the frequency range of $60 \sim 150 \mathrm{kHz}$ was estimated as $4904 \mathrm{~m} / \mathrm{s}$, which was close to the predicted group velocity $(\sim 4962 \mathrm{~m} / \mathrm{s})$ of the $\mathrm{L}(0,1)$ mode. The consistency between the measured data and the group velocity dispersion curve indicated that the proposed squirrel-cage MsS could successfully generate $\mathrm{L}(0,1)$ mode in the tested tube.

\section{Defect Inspection Experiments and Discussion}

The performances of the squirrel-cage MsS in defect detection were experimentally evaluated in a tube with $90^{\circ}$ elbow. The size of the elbowed tube was the same as that in the simulation model, and the MsS was installed onto the upper end of the tube. The artificial notch defect and the through-wall defect were alternatively machined in the small-diameter tube. The operation frequency of the MsS was fixed as $90 \mathrm{kHz}$ in the tests. Figure 7(a) illustrates the paths of UGW transmission and reflection in the defective tube for the convenience of the identification of UGW inspection signals. 


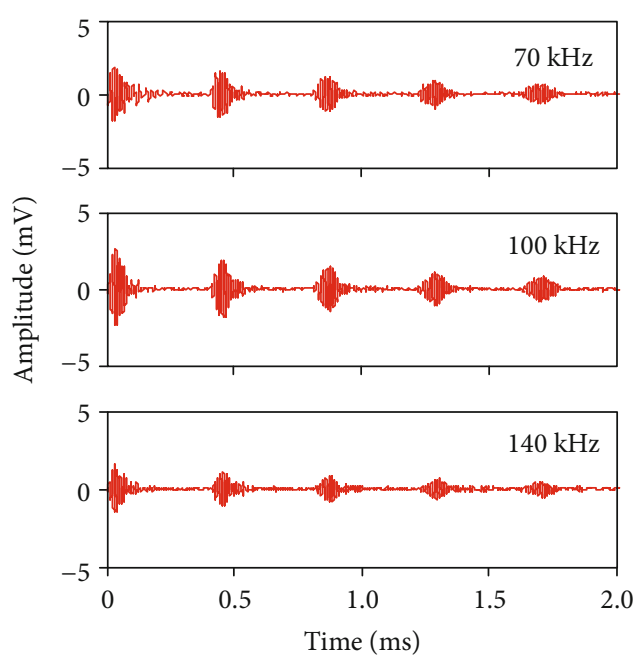

(a)

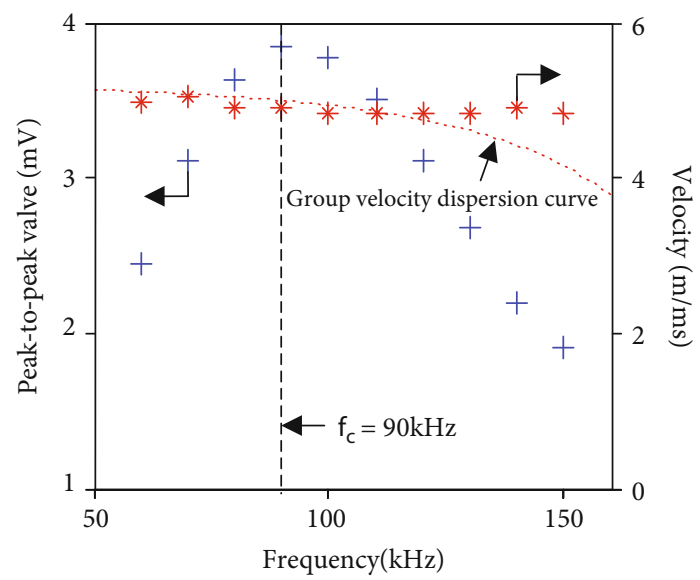

(b)

FIGURE 6: (a) Time-domain UGW signals obtained at different operation frequencies. (b) Relationship between the peak-to-peak amplitude of the first reflection UGWs and the operation frequency.

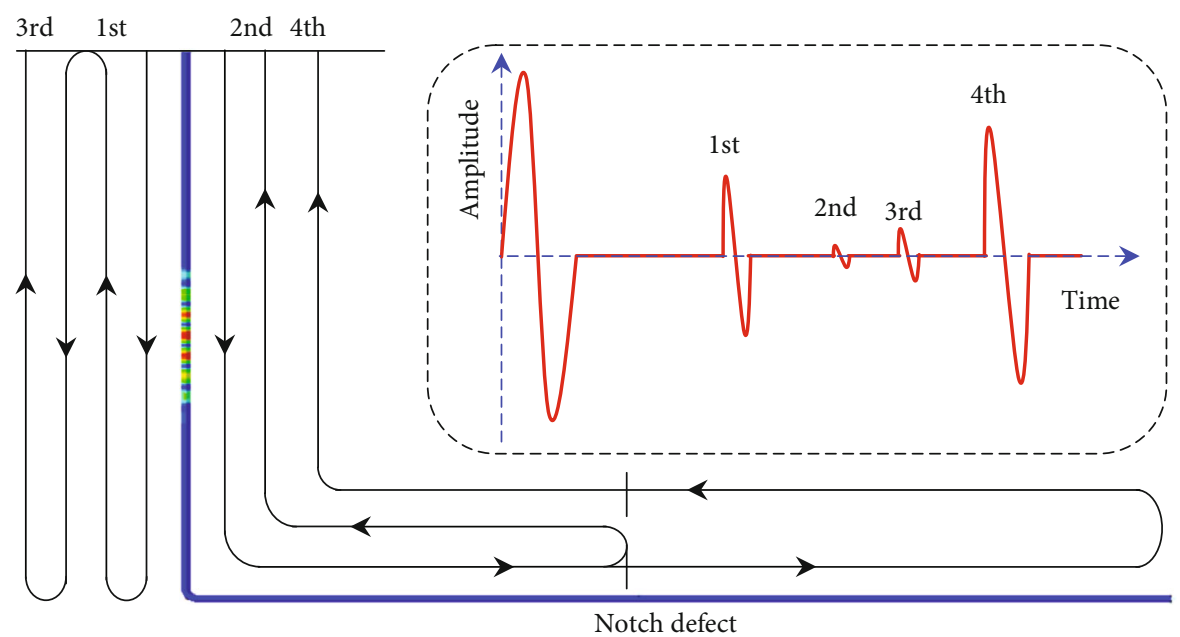

(a)
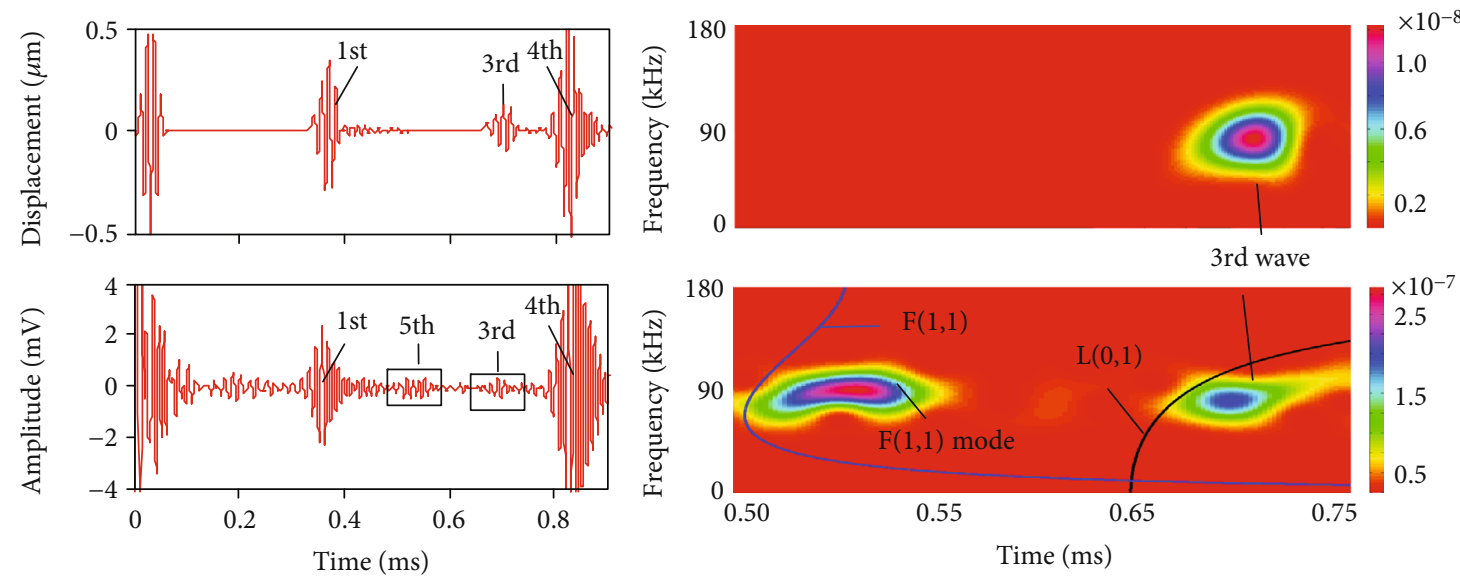

(b)

FIgURe 7: (a) Propagation paths of UGWs in the defective elbowed tube. (b) UGW inspection results obtained from a healthy tube. 

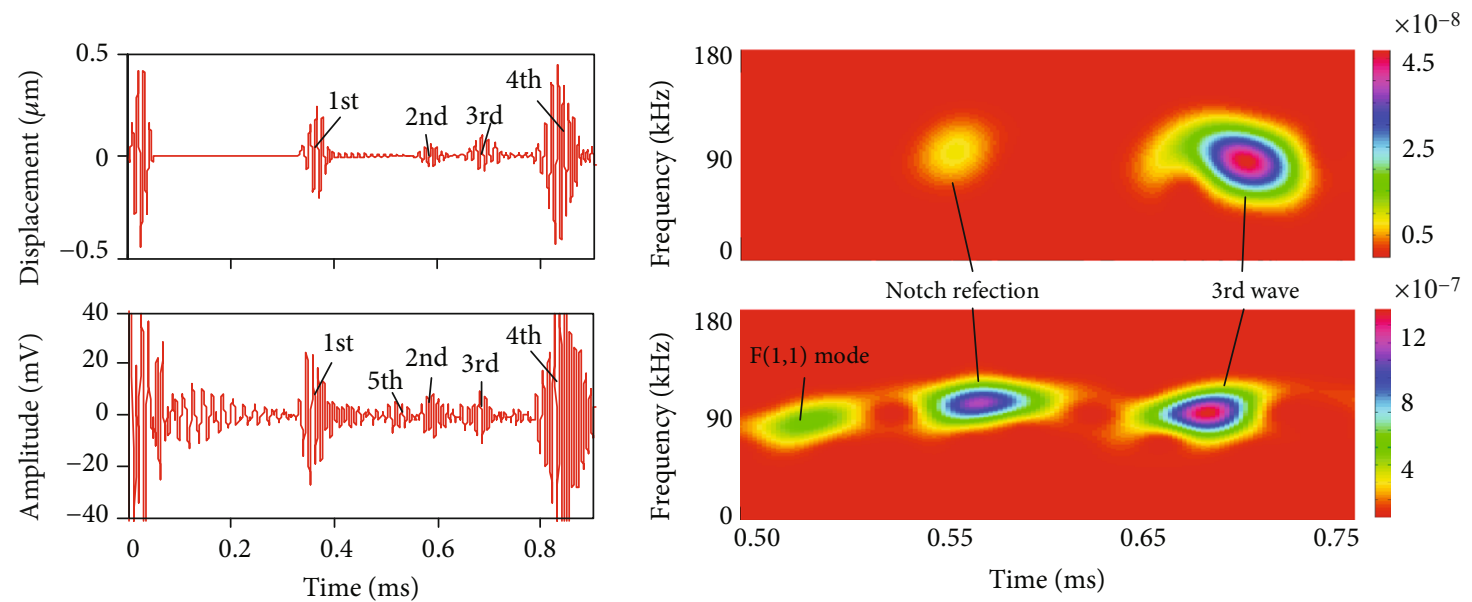

(a)
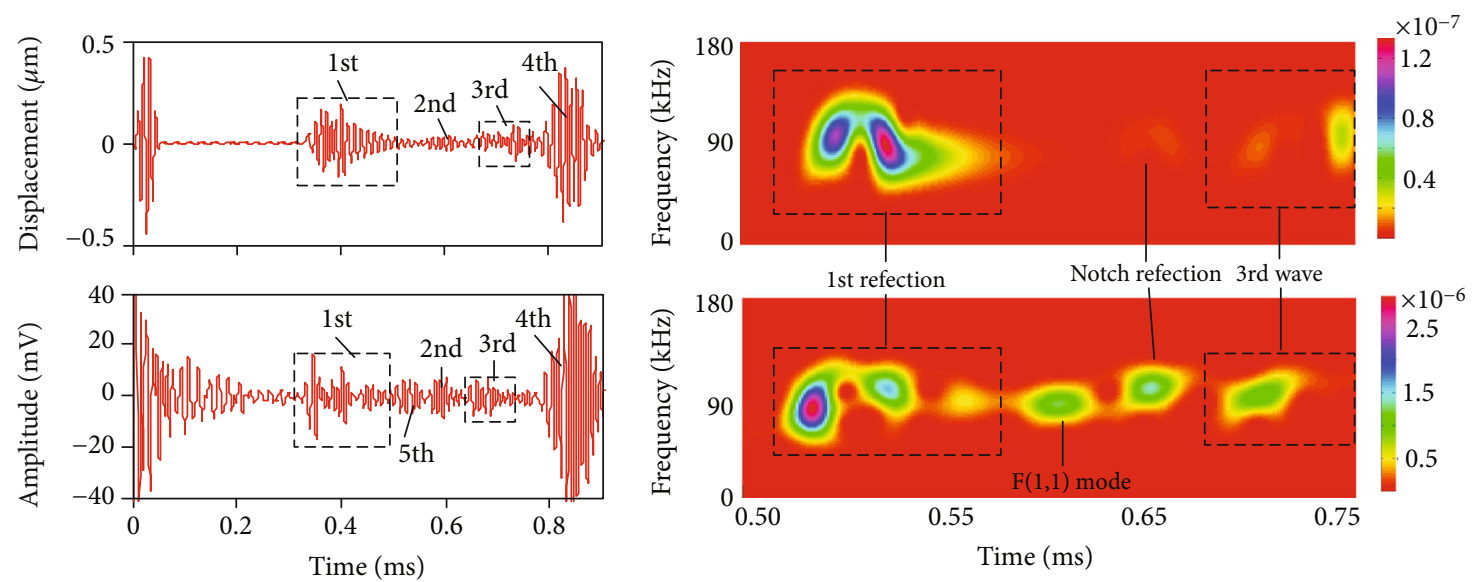

(b)

FIGURE 8: UGW inspection results obtained from a tube with a notch defect in the horizontal straight section (a) and a tube with a throughwall defect at the elbow and a notch defect in the horizontal straight section (b).

The first wave signal (1st wave packet) received by the MsS came from the inherent reflection of the $\mathrm{L}(0,1)$ mode at the elbow region. If the through-wall defect was machined at the elbow region, the defect-induced wave was expected to experience a slight delay in propagation time compared to the 1st wave packet. The waves reflected from the notch defect (referred as the 2nd wave packet) arrived at the MsS location earlier than the second inherent reflection (referred as the 3 rd wave packet) of the $\mathrm{L}(0,1)$ mode at the elbow region. The waves echoed from the far end of the tube came after the 3 rd wave packet. Figures 7(b) and 8 demonstrate the comparisons between the simulation and experimental results. In Figures $7(\mathrm{~b})$ and 8 , the first and second rows, respectively, indicate the simulation and experimental results, whereas the right column presents the short-time Fourier transform (STFT) results of the time-domain signals in the left column.

As a baseline for defect signal identification, the first UGW inspection experiment was conducted on a healthy tube. The $\mathrm{L}(0,1)$ mode experienced stronger reflection at the elbow compared with the simulation result (Figure 7(b)).

It can be observed from the experimental result that the amplitude ratio of the trailing waves to the first reflection wave is relatively higher than that in the simulation result. In the elbow forming process, the actual wall thickness varies along the arc segment of the elbow region. Therefore, it is difficult to accurately model the actual geometrical shape of the elbow. In the simulation, the wall thickness along the arc segment of the elbow region was a constant. We tended to ascribe the mismatching results between the simulated and experimentally measured trailing waves to the differences between the simulated and actual elbows.

In addition, a wave packet (marked as the 5th wave packet) with a small amplitude was observed between the first and second reflection waves of the $\mathrm{L}(0,1)$ mode. Through comparing the dispersion curve with the STFT results, the 5 th wave packet was identified as the reflection of $\mathrm{F}(1,1)$ mode from the elbow. $F(1,1)$ mode was generated at the elbow region due to mode conversions of UGWs (Figure 3(b)). The coupling between the squirrel-cage MsS and the tube surface might differ in the regions covered by the two $\mathrm{C}$-shaped sensor elements. The sensor installed onto the tested tube was not perfectly axisymmetric and might receive the flexural mode.

The indication of the $F(1,1)$ mode can also be found in the experimental signals obtained from defective tubes, as 
shown in Figures 8(a) and 8(b). From the perspective of defect detection, the experimental results in Figure 7 (b) can be used as the baseline for identifying the experimental signals obtained from the tube with defects.

In the case of a notch defect, the signals in the time interval between the 4 th and 5 th wave packets are concerned. The $\mathrm{L}(0,1)$ mode reflected from the notch is displayed as the wave packet at $0.6 \mathrm{~ms}$ (Figure $8(\mathrm{a})$ ). The results were consistent with the simulation results. Figure $8(\mathrm{~b})$ demonstrates the results obtained from the tube with a through-wall defect and a notch defect. As shown in Figure 8(a), similar conclusions can be concluded from both the experimental and simulation results that the through-wall defect leads to the decrease in the amplitude of the first inherent reflection of $\mathrm{L}(0,1)$ mode and the generation of additional wave packet superposed with the 1st wave packet. The experimental results in Figure 8 indicated that the proposed squirrel-cage MsS could generate L $(0,1)$ mode UGW to successfully detect both the through-wall defect in the elbow and the notch defect in the straight section of tube.

\section{Conclusions}

Both finite element simulations and experiments were performed to investigate the ability of $\mathrm{L}(0,1)$ mode guided waves in detecting the defect of steel tubes $(10 \mathrm{~mm}$ in diameter, $2 \mathrm{~mm}$ in wall thickness) with $90^{\circ}$ elbow. When the $\mathrm{L}(0,1)$ mode propagated through the elbow region, $7.4 \%$ of the wave energy was reflected back. Due to mode conversion, the transmitted waves were decomposed into three parts: the $\mathrm{L}(0,1)$ mode, $\mathrm{F}(1,1)$ mode, and $\mathrm{F}(2,1)$ mode. Thus, the influence of the anomalous waves on the defect detection was limited. The $\mathrm{L}(0,1)$ mode guided waves reflected from a healthy elbow were manifested as a single wave packet. However, due to the existence of a through-wall defect at the elbow region, the reflected guided waves were shown as two overlapped wave packets, which could be used as an indicator for defect detection in the elbow.

To generate and receive $\mathrm{L}(0,1)$ mode guided waves in the tested tube, a novel magnetostrictive sensor with squirrelcage elements was adopted. The central frequency of the sensor was measured to be around $90 \mathrm{kHz}$. Experimental results obtained with the squirrel-cage MsS on healthy and defective tubes were consistent with the results of finite element simulation. After short-time Fourier transform, the guided waves inspection signals were used to successfully identify the artificial through-wall defect at the elbow and a notch defect at the straight part of the tube. In future, the coupling between the proposed magnetostrictive sensor and the tube will be improved by using clamp so that unwanted flexural mode can be suppressed. In addition, the ability of the experimental system on defect detection will be experimentally evaluated by introducing even smaller defects to the elbowed region.

\section{Data Availability}

The data used in this paper is available for the all the readers. The readers can write an email to us to get the data files.

\section{Conflicts of Interest}

The authors declare that they have no competing interests.

\section{Acknowledgments}

This study was supported by the National Natural Science Foundation of China (Project No. 11572011) and the International Research Cooperation Seed Fund of Beijing University of Technology (Project No. 2018A07).

\section{References}

[1] H. H. Yin, H. Chen, and Z. B. Peng, "Application of SOM network in ship pipeline leakage detecting," Advanced Materials Research, vol. 468, pp. 538-541, 2012.

[2] A. Teitsma, S. Takach, J. Maupin, J. Fox, P. Shuttleworth, and P. Seger, "Small diameter remote field eddy current inspection for unpiggable pipelines," Journal of Pressure Vessel Technology, vol. 127, no. 3, pp. 269-273, 2005.

[3] S. Thirunavukkarasu, B. P. C. Rao, S. Vaidyanathan, T. Jayakumar, and B. Raj, "Influence of sodium deposits in steam generator tubes on remote field eddy current signals," International Journal of Pressure Vessels and Piping, vol. 85, no. 4, pp. 211-218, 2008.

[4] W. S. Singh, C. K. Mukhopadhyay, and B. P. C. Rao, "Development of a high sensitive magnetic flux leakage instrument for imaging of localised flaws in small diameter ferromagnetic steel tubes," IET Science, Measurement \& Technology, vol. 12, no. 7, pp. 932-936, 2018.

[5] Y. Kang, J. Wu, and Y. Sun, "The use of magnetic flux leakage testing method and apparatus for steel pipe," Materials Evaluation, vol. 70, no. 7, pp. 821-827, 2012.

[6] V. Suresh, A. Abudhahir, and J. Daniel, "Development of magnetic flux leakage measuring system for detection of defect in small diameter steam generator tube," Measurement, vol. 95, pp. 273-279, 2017.

[7] M. Wang, D. Han, and S. Li, "The application and research of high-frequency ultrasonic reflection technique used in the measurement of small diameter's tube cavity size," Measurement, vol. 46, no. 1, pp. 521-526, 2013.

[8] M. Bernal, I. Nenadic, M. W. Urban, and J. F. Greenleaf, "Material property estimation for tubes and arteries using ultrasound radiation force and analysis of propagating modes," The Journal of the Acoustical Society of America, vol. 129, no. 3, pp. 1344-1354, 2011.

[9] J. Davies and P. Cawley, "The application of synthetic focusing for imaging crack-like defects in pipelines using guided waves," IEEE Transactions on Ultrasonics, Ferroelectrics, and Frequency Control, vol. 56, no. 4, pp. 759-771, 2009.

[10] A. C. Cobb, H. Kwun, L. Caseres, and G. Janega, "Torsional guided wave attenuation in piping from coating, temperature, and large-area corrosion," NDT \& E International, vol. 47, pp. 163-170, 2012.

[11] Z. Liu, Q. Xu, Y. Gong, C. He, and B. Wu, "A new multichannel time reversal focusing method for circumferential lamb waves and its applications for defect detection in thickwalled pipe with large-diameter," Ultrasonics, vol. 54, no. 7, pp. 1967-1976, 2014.

[12] T. Yamamoto, T. Furukawa, and H. Nishino, "Frequency dependence of the defect sensitivity of guided wave testing 
for efficient defect detection at pipe elbows," Materials Transactions, vol. 57, no. 3, pp. 397-403, 2016.

[13] H. Nishino, S. Masuda, Y. Mizobuchi, T. Asano, and K. Yoshida, "Long-range testing of welded elbow pipe using the T $(0,1)$ mode ultrasonic guided wave," Japanese Journal of Applied Physics, vol. 49, no. 11, article 116602, 2010.

[14] B. Pavlakovic and M. Lowe, Disperse User's Manual Version 2.0. 11, Imperial College, University of London, 2001.

[15] X. Zhang, Z. Tang, F. Lv, and X. Pan, "Helical comb magnetostrictive patch transducers for inspecting spiral welded pipes using flexural guided waves," Ultrasonics, vol. 74, pp. 1-10, 2017.

[16] X. Liu, B. Wu, and C. He, "Megahertz-range longitudinal guided wave excitation by a flexible magnetostrictive sensor for steel strand inspection," International Journal of Mechatronics and Automation, vol. 2, no. 3, pp. 217-226, 2012.

[17] C. Aristégui, P. Cawley, and M. Lowe, "Reflection and mode conversion of guided waves at bends in pipes," AIP Conference Proceedings, vol. 509, no. 1, pp. 209-216, 2000.

[18] M. Qi, S. Zhou, J. Ni, and Y. Li, "Investigation on ultrasonic guided waves propagation in elbow pipe," International Journal of Pressure Vessels and Piping, vol. 139-140, pp. 250-255, 2016.

[19] L. Laguerre, J. C. Aime, and M. Brissaud, "Magnetostrictive pulse-echo device for non-destructive evaluation of cylindrical steel materials using longitudinal guided waves," Ultrasonics, vol. 39, no. 7, pp. 503-514, 2002.

[20] J. Xu, Y. Sun, M. Cong, and X. Wu, "A feasibility study of tension measurement using longitudinal mode guided waves based on the magnetostrictive effect," International Journal of Applied Electromagnetics and Mechanics, vol. 52, no. 1-2, pp. 391-397, 2016.

[21] S. H. Cho, C. I. Park, and Y. Y. Kim, "Effects of the orientation of magnetostrictive nickel strip on torsional wave transduction efficiency of cylindrical waveguides," Applied Physics Letters, vol. 86, no. 24, article 244101, 2005.

[22] H. Kwun, S. Y. Kim, and G. M. Light, "Magnetostrictive sensor technology for long-range guided wave inspection and monitoring of pipe," The NDT Technician, vol. 10, no. 2, pp. 6-9, 2011.

[23] S. Vinogradov, G. Light, T. Eason, and M. G. Lozev, “Mockup evaluation of magnetostrictive transducers for guided wave monitoring of pipe at $200^{\circ} \mathrm{C}$," in 26th ASNT Research Symposium, pp. 259-266, 2017.

[24] B. Yoo, S. M. Na, A. B. Flatau, and D. J. Pines, "Ultrasonic guided wave sensing performance of a magnetostrictive transducer using Galfenol flakes-polymer composite patches," Journal of Applied Physics, vol. 117, no. 17, article 17A916, 2015.

[25] S. A. Vinogradov, "Magnetostrictive transducer for torsional guided waves in pipes and plates," Materials Evaluation, vol. 67, no. 3, pp. 333-341, 2009.

[26] S. H. Cho, H. W. Kim, and Y. Y. Kim, "Megahertz-range guided pure torsional wave transduction and experiments using a magnetostrictive transducer," IEEE Transactions on Ultrasonics, Ferroelectrics, and Frequency Control, vol. 57, no. 5, pp. 1225-1229, 2010. 


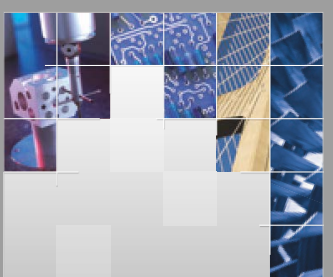

\section{Enfincering}
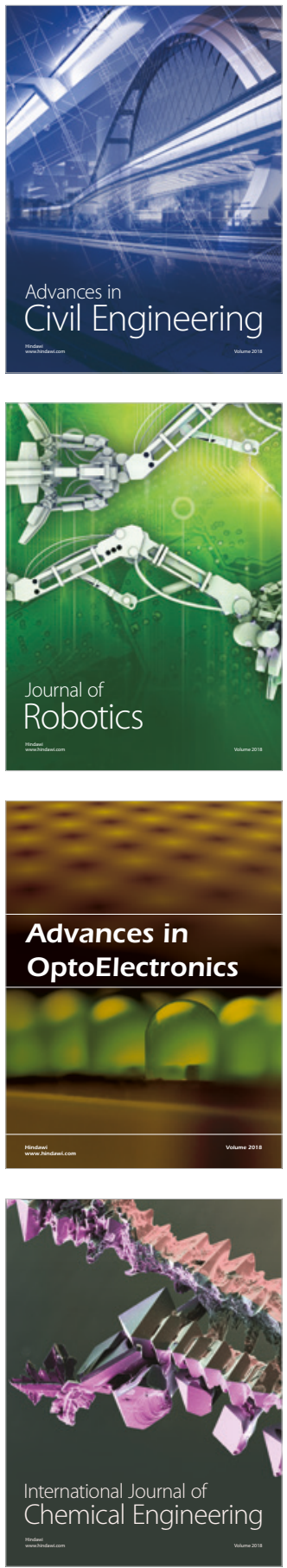

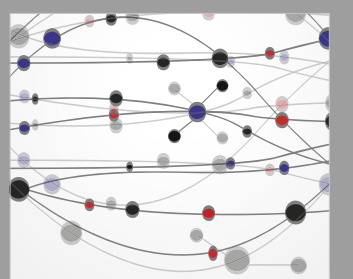

\section{Rotating \\ Machinery}

The Scientific World Journal

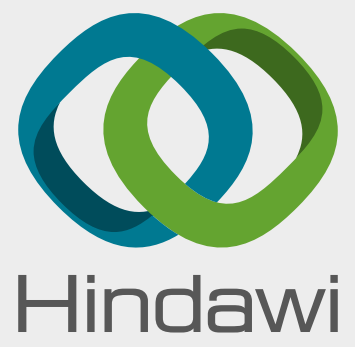

Submit your manuscripts at

www.hindawi.com
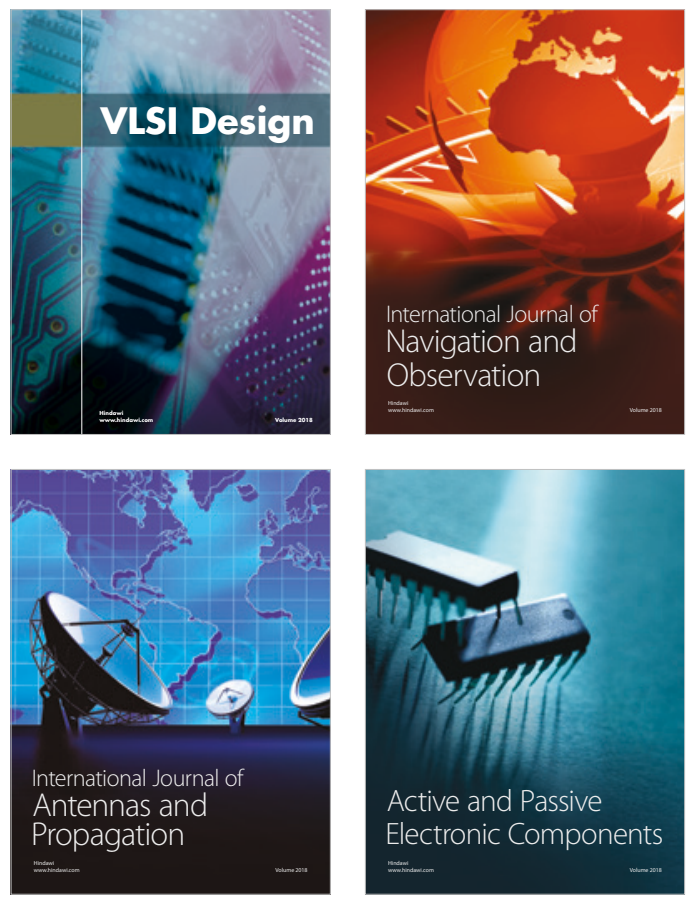
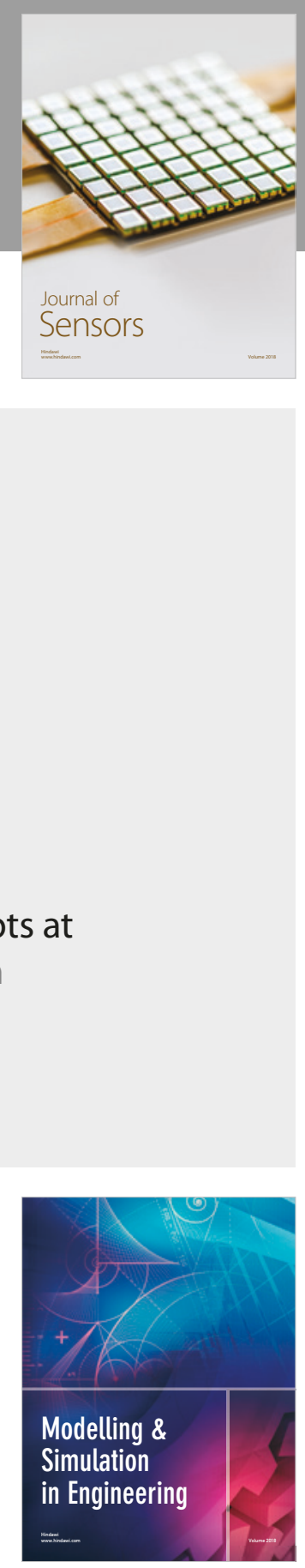

\section{Advances \\ Multimedia}
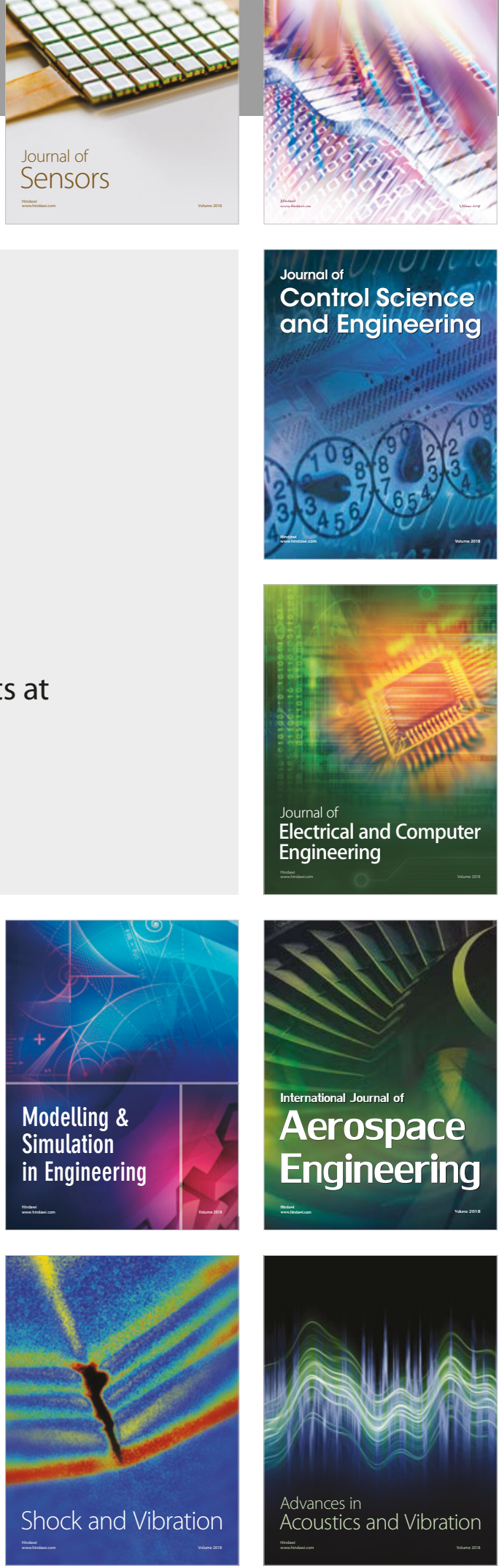